related illnesses or acquired disabilities is a complex task. However, it is vital that policy-makers be aware of the scope and scale of poor or non OSH in order to implement effective measures in this policy area. If we do not value life and health impacts, we will implicitly make a trade-off or these values may get even assigned zero-weight.

Methods The first phase of the large-scale study commissioned by EU-OSHA in 2015, consisting of an identification and assessment of the available data relevant to costing models that is available in each of the Member States. We concluded that the development of a comprehensive, comparable estimate covering all EU member states would not be feasible at present due to the lack of data at national level. Therefore it was decided that the second phase of the project would consist of two strands.

Results First, EU-OSHA collaborated in the development of updated ILO estimates, based on available data at international level, to calculate an approximate cost estimation for each EU member state including Norway and Iceland. The findings reveal that work-related injury and illness result in the loss of $3.7 \%$ of GDP globally (EU28 3.1\%), at an annual cost of roughly $€ 2.206$ billion (EU28 $€ 446$ billion) Second we commissioned research to develop a comprehensive cost estimate for five member states, where sufficient data for such a calculation is available. These results can also be used to validate and refine the ILO estimates for the European level.

Discussion The results confirm earlier research that a high level of prevention contributes to the productivity and wealth of a country. Lower prevention results in higher costs to society

\section{MODERN WAYS OF PROMOTING SAFETY MESSAGES}

Jawed Ahmad Mangi*. Saudi Electricity Company, Riyadh, Saudi Arabia

\subsection{6/oemed-2018-ICOHabstracts.76}

Introduction Increasing number of accidents and incidents in organisation each year which included fatalities, serious injuries have had a great impact on the production, efficiency, reputation, progress and financial reports of the company.

The problem is the people and the task is to change the culture. Make them realise to work safely at their own, even if not supervised.

Now the businesses are adopting a new safety culture by using digital practices to reach their employees via digital displays, desktop communications and handheld devices through which the real time messages could be transferred to the work force to take timely a safe action in the field on dangerous and hazardous material and equipment.

This writing provides a summary of efforts for the best modern practices applied for messaging for the company employees. It is applied in an Electricity distribution company.

During its operational and maintenance activities where daily risk associated jobs are carried out by the workers, miles away from office desk in the field and it also applies to the office employees too.

The paper concludes with some suggestions and adoption of best practices of safety messages promotion for on job accidents' prevention.

Method Various meetings were held in this regard on top, middle and at lower management level, many discussions done, surveys conducted, interviews done from the workers and opinions sought from the field and office staff too.
Discussion and result After discussions and interviews, meetings, it was decided that along with other tactics, also the services of the software development companies required to be hired to design a system of our ideas for the communication and promotion of safety messages to our staff in real time.

\section{WORK ACCIDENTS/INJURIES REGISTERED IN IRAQ FOR THE YEAR 2016}

Imad Abdulkhaleq Sabre. Iraqi NCOHS - MLSA

\subsection{6/oemed-2018-ICOHabstracts.77}

Introduction The existing legislation for occupational accidents diseases and injuries did not made us gain real information about the magnitude of the problem in Iraq.

Therefore we implemented a new way for registration of occupational injuries through the cooperation between the CIS centre/NCOSH/MoLSA/ and the planning directorate $/ \mathrm{MoH}$ in Iraq, since 2012, so that build and gain basic informations about the epidemiology of the problem, to be able to improve the national planes to reduce and trying to stop them.

Methods The emergency clinics in all Iraqi governorate hospitals filled up a questionnaire paper prepared for this purpose and then sent them to the $\mathrm{MoH}$ and then to the NCOSH CIS centre, where a special electronic program was prepared to enter the informations of the questionnaire papers, then studied statically and gain the needed results.

Results and discussion The yearly numbers of the registered cased according to the legislations and since more than 40 years was around 200 cases only. While according to the new program the number became between 4000 to 7000 cases each year for the last 5 years.

$96.74 \%$ of the registered cases were male worker.

$49.5 \%$ of the age group $15-29$ years, while $36.8 \%$ of the age group

$30-44$ years of age.

$76.4 \%$ of the work injuries happened to workers in the private sector.

$81.4 \%$ happened in the work place while $18.9 \%$ were commuting accidents.

$67.1 \%$ happened during morning work shifts.

$30.9 \%$ caused by machines and instruments, and $21.9 \%$ because of falls.

$18.4 \%$ affected the workers heads, $18.1 \%$ the left upper limbs and $17.4 \%$ affected the right upper limbs.

2014 registered work accidents caused wounds to the injured workers, 1135 caused only bruises and scratches, and 601 fractures to the affected workers.

The total registered number of workers deaths was 17 cases during the year 2017.

$41.1 \%$ of them were from governmental sector, $47 \%$ were because of terrorists, $23.5 \%$ because of electric shocks and also $23.5 \%$ because of lifting and transport media while $5.8 \%$ because of exposure to chemicals.

Aim Gaining and building the basic information about the magnitude and epidemiology of work accidents and injuries in Iraq, so that to improve the national planes to reduce and trying to stop them.

Objectives

- To increase the scope and effectiveness of the investigation of causes of occupational accidents, and the identification and implementation of preventive measures. 